\title{
Impact of Artificial Intelligence in Special Need Education to Promote Inclusive Pedagogy
}

\author{
Shalini Garg and Shipra Sharma
}

\begin{abstract}
Artificial Intelligence (AI) and technology has already touched the life of individuals, more generally, it has influenced educational sector to make it more inclusive and accessible for students with visual, hearing, mobility and intellectual disabilities. The use of AI has not only impacted students with special needs, but has also impacted educational institutions in creating inclusive pedagogies. The present study is a working paper that has tried to analyze how AI has impacted education for students with special needs. The data collection was based on qualitative research that was conducted using focused interviews from teachers and students with special needs. The data was also collected through literature present in the academic databases EBSCO that comprised of Web of Science, Scopus and Science Direct, newspapers, magazines, blogs. The responses obtained were analyzed using Content Analysis. The study in particular focused on whether the literature covered the theme of analyzing the impact of AI on (a) Special Need Education; (b) AI helping teachers to promote special need education. The study also tried to propose the framework for an inclusive future of Special Need Education based on focused interview.
\end{abstract}

Index Terms -Disabilities, AI, special need education.

\section{INTRODUCTION}

Today, due to disruption in technology lot of new things like -work automation, IOT, AI, machine learning AR, VR, etc. are changing the workplace, especially for people with disabilities and demanding more agility in workforce to adopt faster (changes in ways of working and culture) and create inclusive and accessible organizations.

In many developing countries of Asia and the Pacific, people with disabilities are commonly trapped in an atrocious cycle of exclusion from society, participation in jobs and mainstream programs for development.The World report on disabilities by World Health Organization (WHO) [1] and Laabidi [2] indicates that more than one billion people in the world suffer from some or the other disabilities. Such people lack healthcare, education and job opportunities [3]. Without convenient assistive devices, they often lack the means to engage in education for living independent and better life. As per an estimation by WHO, there are more than 1 billion people who would benefit from one or more assistive devices or products.

Microsoft's Annual Report [4] (letter to Shareholders), has emphasized on the impact of technology on each and every aspect of life, work and society at large. It has indicated that

Manuscript received December 23, 2019; revised March 11, 2020.

Shalini Garg and Shipra Sharma are with GGSIP University, Sector-16C, Dwarka, Delhi, India (e-mail: shalinivineet@yahoo.com, shiprasharma_1@rediffmail.com). the world needs a trusted technology that can benefit people and society. The technology platforms and tools can enable the creativity in people with and without disabilities. The advancement in technologies has enabled Microsoft to empower people with disabilities, for instance, it has provided learning tools that has helped children with dyslexia to learn more. This has broadened the scope of AI and Machine Learning. AI Technology has been developed for performing high-computing tasks with the help of computer tools for the ease of human [5]. Although the machines can never replace a human being but can assist them for better work arrangements. Recent developments in AI may enable the development in the education and learning sector to empower people with special needs in education.

\section{A. Disabilities}

As per WHO, 2011 [1] Disability is the human condition. Almost everyone suffers from it either temporarily or permanently. It has been defined in numerous manners, The International Classification of Functioning, Disability and Health (ICF) emphasizes on environmental factors in creating disability. ICF has classified into Impairment, Disabilities and Handicaps. The United Nations defines Impairment and Disability differently:

Impairment: "Any loss or abnormality of psychological or anatomical structure or function".

Disability: "Any restriction or lack (resulting from an impairment) of ability to perform an activity in the manner or within the range considered normal for a human being".

People with Disabilities are also referred as People with special needs [6]. Over the past few years, the term special needs have been popularly used as a synonym for disability [7]. Rather than identifying anyone as having a certain disability, the person is referred as having special needs. As per White Paper on Rights of Persons with Disabilities [8], "disability is imposed by society when a person with a physical, psychosocial, intellectual, neurological and/or sensory impairment is denied access to full participation in all aspects of life, and when society fails to uphold the rights and specific needs of individuals with impairments". According to this report persons with disabilities normally face three kinds of interrelated barriers: social, psychological and structural (that is infrastructure and operations).

According to the research by Florida Department of Education, (2010) disabilities are categorized as Specific Learning/Language Disabilities, Hearing, Visual and Physical Disabilities. As per other researchers, [2] persons with disabilities are also grouped into four groups: Mobility Impairment, Hearing Impairment, Visual Impairment and Cognitive Impairment. AI provides different technologies to adapt to the requirements of people with disabilities. 


\section{LITERATURE REVIEW}

\section{A. AI and Special Need Education}

Various researches have taken place in the area of AI (AI). It is usually defined as "the mechanical simulation system of collecting knowledge and information and processing intelligence of universe: (collating and interpreting) and disseminating it to the eligible in the form of actionable intelligence" [9]. It is composed of information agents that can be either in the physical form as devices (such as Robots or can be virtual such as software. According to Morrison [10], as AI matures, it becomes increasingly important to understand the kind of things that people with disability would require as a part of their technology tool kit. The benefits of AI have been acknowledged in education; however, the research fraternity has started exploring its benefits for people with special needs in education [5]. AI and Special need Education collaborate together to enable development of individuals suffering from disabilities. Students with learning, hearing, visual and mobility impairment can seek benefits with the use of Artificial Intelligence in education. The research study has also indicated AI as one of the assistive technology for PwDs. As per an article by Lynch [2], AI has provided around-the-clock care using Robotics for people with disabilities. AI has helped the people to use mobile applications without even clicking on it. For instance "Siri" in mobiles has enabled people to access mobile applications without even actually clicking actually clicking on them. Another example from Google "Alexa" that has enabled people to ask for any information without typing on the search bar. Both these applications (Siri as well as Alexa) work on AI (speech recognition. Such applications can provide assistive services to people.

This paper tries to investigate the impact of AI on special need students and the assistance these tools can extend to teachers in evaluating and imparting education as per the requirements of students with special needs.

\section{B. Specific Learning/Language Disabilities}

In every institution each and every student requires some or the other assistance at their level in reading, writing, pronouncing and understanding. The level of learning and language disabilities are required to be identified and diagnosed at the tender age of a child [5]. Although there are manual techniques to diagnose learning and language disabilities, however, they can be tackled by machine learning algorithms as expert systems that can be fed to diagnose such disabilities [11]. Various computers based tools as educational technologies has been development to manage the children with dyslexia; these tools include 'Phonological Awareness Educational Software' (PHAES) it facilitates the reader with Phonological awareness. They come as software that can be easily navigated and can be understood with the help of simple graphics without cognitive overload [5]. Robotic assistance is been implemented for managing the children suffering from Autism Spectrum Disorder (ASD) so that they can learn social skills understand the emotions [12]. For instance, British Primary school used Humanoid Robots for children suffering from ASD to understand Human Emotional State [13]. A game called LIFEisGAME is developed for kids with ASD which helps them to understand facial expressions, also an e-learning tool called Smart Tutoring Model made for children suffering from learning disabilities such that they could understand in an easy and better way [13]. Prentzas [10], Kim Y-D, Hong J-W, Kang W-S, Baek S-S, Lee H-S, An J [14] research results have indicated that robots can be used as an assistive tool for people with disabilities, the research has also indicated that robot-assisted observation systems are used for children with autism in kindergarten for observing their development. Artificial Neural Networks and Fuzzy Logic can be used for diagnosis and accessing the children suffering from ASD, the pseudo algorithm is applied to access the autism disorder [5]. Microsoft provides an AI tool, Equadex for children with learning disabilities and mainly for children with Autism, Equadex provides a mobile navigated environment to easily communicate and understand through visual presentations (Equadex, Microsoft Cognitive Services, 2017) [15]. AI is also utilized in the diagnosis process for instance web-based system based on deep learning is used for accessing the children with autism and learning disabilities that send reports to the medical officer and parents.

\section{Hearing Impairment}

Microsoft comes with Microsoft Translator as an AI assistive device for deaf learners. The device comes with a headset worn by the speaker that translates speech signals into captions that are visible to the learner with hearing impairment. The translator provides the translation in 60 different languages, giving an ease for deaf learners to understand (Roach, [16], Sam McNeill, [17]). Zeng, Fan-Gang, [18] Over the Hearing aid (OTC) uses Deep learning and AI technologies such as virtual and augmented reality that improves the performance even of normal-hearing people. An intelligent hearing aid will offer a personalized and interactive hearing experience like sound scene analysis, sound protection and warning and even real-time language translation.

\section{Visual Impairment and Physical Impairment}

Children with visual impairment take a while to accommodate in a mainstream classroom, but it is not impossible. Such students require different set of visual devices such that they feel included in a classroom. Mboshi [19] suggests classroom teachers to adopt unique teaching ways for effective and inclusive classroom setting. Visually impairment may include totally blind or low-vision impairment, for any kind of visual impairment, visual devices (such as Braille, change in text size, color etc.) available to give such children an access to education and feel included.

Learner's with physical impairment requires support in availing educational services which helps them to maintain their autonomy during the educational course.

\section{Methodology}

The study is exploratory in nature and has used qualitative research method to gather information. The research has been 
conducted using focused interviews. The responses were obtained from teachers as well as students with disabilities. The sample of focused interviews consisted of ten students with disabilities and five teachers teaching children with special needs (as shown in Table I). The sample was selected, in order to understand the support provided by institution and strategies taken up by teachers to promote inclusive learning environment. The responses were analyzed using Content Analysis that helped in suggesting an inclusive pedagogy and teaching style to promote special need education.

\section{A. Demographical Profile}

TABLE I: DEMOGRAPHICAL PROFILE OF RESPONDENTS

\begin{tabular}{|c|c|c|}
\hline Variables & Description & Frequency \\
\hline \multicolumn{3}{|l|}{ Student } \\
\hline \multirow[t]{2}{*}{ Age } & Between 10 to 12 years & \multirow{2}{*}{$\begin{array}{l}4 \\
6\end{array}$} \\
\hline & Between 13 to 15 years & \\
\hline \multirow[t]{2}{*}{ Gender } & Male & 10 \\
\hline & Female & Nil \\
\hline \multirow[t]{6}{*}{ Education } & Class 5th & 2 \\
\hline & Class 6th & 1 \\
\hline & Class 7th & 1 \\
\hline & Class 8th & 1 \\
\hline & Class 9th & 2 \\
\hline & Class $10^{\text {th }}$ & \\
\hline \multirow[t]{4}{*}{ Disabilities } & Learning Disability & 3 \\
\hline & & 1 \\
\hline & Hearing Disability & $\begin{array}{l}2 \\
4\end{array}$ \\
\hline & Physical Disability & \\
\hline \multicolumn{3}{|l|}{ Teacher } \\
\hline \multirow[t]{3}{*}{ Age } & Below 30 Years & 1 \\
\hline & $31-40$ years & 3 \\
\hline & Above 40 years & 1 \\
\hline \multirow[t]{2}{*}{ Gender } & Male & Nil \\
\hline & Female & 5 \\
\hline
\end{tabular}

\section{B. Instrumentation}

The responses from the target groups were collected through focused interviews. The interview was primarily conducted in two steps:

In the first step, the interview was conducted with the teachers teaching students with special needs, so as to understand the teaching style and institutional support to promote inclusive pedagogy.

In the second step, the interview was conducted with the students with disabilities for understanding problems faced by students with special needs and if they are addressed by institution or teacher.

The questions asked during focused interview to the respondents were as shown in Table II:

TABLE II: QUESTIONS ASKED DURING FOCUSED INTERVIEW

\begin{tabular}{lll}
\hline \hline Questions & & \\
\hline Respondents: Teachers & $\begin{array}{l}\text { Respondents: Students with } \\
\text { Disabilities }\end{array}$ \\
\hline 1. How can a teacher provide & $\begin{array}{l}\text { 1. Do you feel happy in the school } \\
\text { a supportive } \quad \text { learning in the class? }\end{array}$ \\
\hline \hline
\end{tabular}

environment for students with special needs?

2. How do you manage a class having mix of students with or without disabilities?

3. How do you get institutional support to promote inclusive learning environment?

4. Does institution provide reasonable accommodation and technological support to promote inclusive environment?

2. Do you feel rejection in the class? 3. Do you need special assistance for travelling in the campus? If yes, is it provided?

4. What special arrangements are provided during attending lectures?

5. Do you need assistance or special equipment while studying?

6. Do you feel any activity in your study programme that you cannot attend? (n)

\section{DATA ANALYSIS}

The content analysis were performed on the responses obtained from respondents that helped in recognizing the problems associated with children and helped in analyzing the best way of promoting Inclusive Education.

\section{A. Inclusive Future of Special Need Education}

'Education to All' is the fundamental right of every individual being a child or an adult, no individual can be denied by anyone from seeking their right to Education irrespective of their abilities or disabilities. The primary change is required in the education system so as to make the education inclusive to all. Labeling a child as 'visually/ hearing/ learning/ physically impaired children' or 'special child' may lower the education system or education imparted to them. This not only separates the child from the mass but also excludes them from other important stages of social development.

The inclusive education refers to the design of school, classrooms, teaching aids, teaching style and activities to accommodate all students so that all can learn and participate equitably. The focused interview with teachers indicated that in order to promote inclusive education, school needs to understand problems associated with the children. The responses from children also indicated the similar problems (as shown in Fig. 1) faced by them in a classroom.

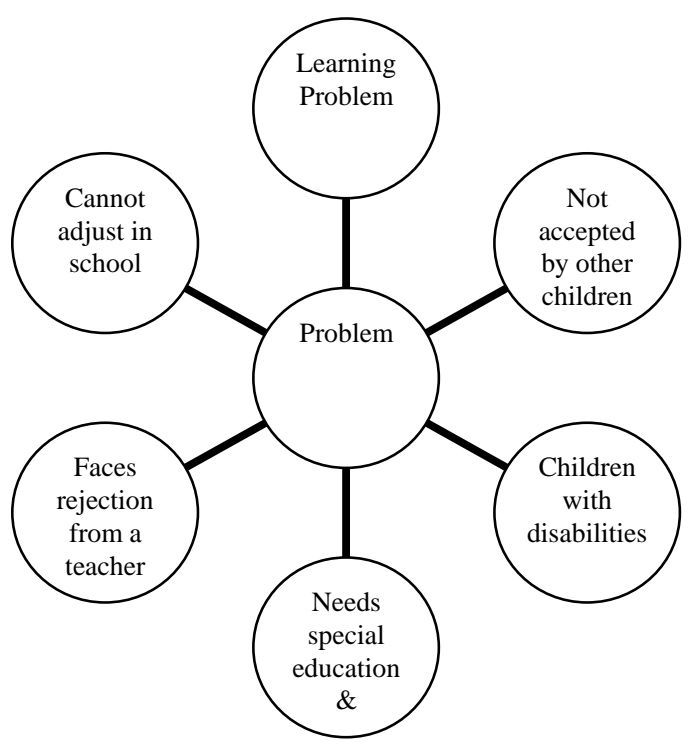

Fig. 1. Problems associated with child. 


\section{B. Problem Associated to Children}

The responses indicated that most of the time, every child whether abled or disabled faces certain problems in the school that may be associated to their learning abilities, not accepted or excluded by other children, rejection from the teacher, cannot adjust to school, needs special education and equipments and children facing disabilities (as shown in Fig. 1). These problems are needed to be addressed by curriculum for promoting Special Need Education and therefore, such curriculum's are not only associated to children with disabilities but requires to accommodate every child, helping them in copping up with their problems.

These problems associated to every child can guide institution to develop Special Need Education and institutionize the Pedagogical changes that can promote Inclusive Education. The curriculum needs to be designed considering all such requirements of the child and the special equipments to make the learning process much easy and enjoyable to every child not necessary 'Children with Disabilities' such that no child feels rejection from teacher or other children.

\section{Promoting Inclusive Education}

Pedagogy is referred as 'the act of teaching together with its attendant's disclosure' (UNICEF, 2014) [20]. It describes what one needs to understand and what skills one needs to inculcate. Fig. 2 represents the Pedagogical requirements and teaching styles to promote inclusive education, this was developed based on the responses obtained from focused interview with teachers and students. The figure represents the combination of low and high Inclusive Teaching Style and Inclusive Pedagogy in the four quadrants, each representing four different forms of Inclusive-Exclusive Education.

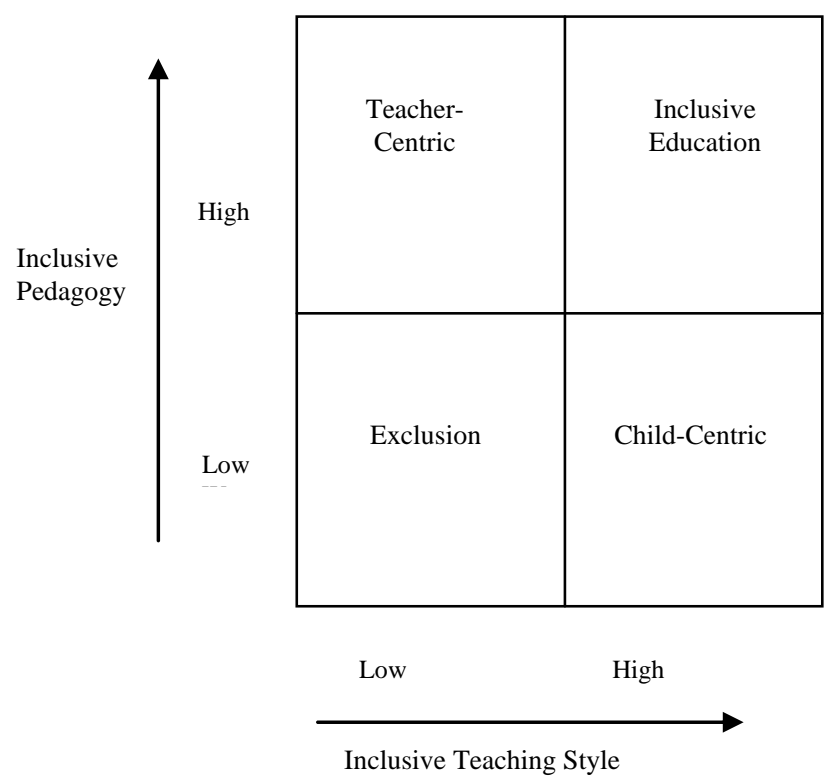

Fig. 2. Inclusive pedagogy and teaching style to promote inclusive education.

Teaching style represents the style exhibited by a teacher to impart education with equal focus towards the needs of a student irrespective of his or her abilities or disabilities whereas, Inclusive Pedagogy refers to that act of teaching individuals, paying attention to their learning requirements, abilities and disabilities.

Exclusion: Low Inclusive Teaching Style and low Inclusive Pedagogy results into exclusion, this indicates that neither the teaching style nor the pedagogy is concerned towards equitable education or special need education. The teacher may only come to deliver the class without taking care of children's needs for learning. Such teachers may categorize the students on the basis of their varied backgrounds and believe that students facing problem may need specially training teachers and staff to educate them.

Teacher-Centric: Low Inclusive Teaching Style and high Inclusive Pedagogy, this style is referred as teacher-centric as it majorly depends on the teacher to whom he or she will pay more attention. Teacher paying attention to specific child may lead to segregation of the children from other class which also does not help the schools to promote equitable education.

Child-Centric: High Inclusive Teaching Style and Low Inclusive Pedagogy also do not lead to inclusive education. Although teacher have high concern for all the students and may opt for different pedagogical style to accommodated every individual. This may further lead to categorization of children as able or disable.

Inclusive Education: High Inclusive Teaching Style and High Inclusive Pedagogy helps in promoting equitable education to all students without categorization depending on their varied backgrounds. This majorly depends implementing changes in teacher's classroom behavior which implies to the way a teacher stands, interact and deliver education. The teacher needs to develop pedagogies that can include every child without discriminations or categorization. The teacher needs to develop pedagogies that make children feel safe and free to exchange their ideas, conversations with each other. The pedagogies are designed in a way that promotes creativity.

In order to promote Inclusive Education, this requires organizational support by implementing inclusive infrastructures with the help of AI Technologies and Augmented Technology to promote Special Need Education.

\section{CONCLUSION}

The study explored that AI Technologies are touching the lives of every individuals and making life easier for children with special needs. Its major impact can be experienced in the area of education where institutions, teachers and parents' tries to promote inclusive education with assistive technologies that can substitute human effort and promote education without discrimination.

The study also suggested implementing inclusive pedagogy that includes every child without categorization. It also suggests developing pedagogies that promotes creativity and will help in creating a safe environment for children where they are free to exchange ideas, conversations with each other and respect the differences in each individual.

\section{CONFLICT OF INTEREST}

There is no potential conflict of interest, as the research 
was not sponsored or funded by any third party.

\section{AUTHOR CONTRIBUTIONS}

Both the authors have contributed to this paper.

\section{REFERENCES}

[1] WHO. (2011). World Report on Disability. [Online]. Available: https://www.who.int/disabilities/world_report/2011/report.pdf

[2] M. Laabidi, M. Jemni, L. J. B. Ayed, H. B. Brahim, and A. B. Jemaa, "Learning technologies for people with disabilities," Research Laboratory of Technologies of Information and Communication \& Electrical Engineering LaTICE, National Higher School of Engineering of Tunis, 2013.

[3] UNESCO Global Report: Opening New Avenues for Empowerment: ICTs to Access Information and Knowledge for Persons with Disabilities, United Nations Educational, Scientific and Cultural Organization (UNESCO), 2013.

[4] Microsoft annual report. (2017). Letter to Shareholders. [Online]. Available: https://www.microsoft.com/investor/reports/ar17/index.html\#

[5] A. S. Drigas and R.-E. Ioannidou, "Artificial intelligence in special education: A decade review," International Journal of Engineering Education, vol. 28, no. 6, pp. 1366-1372, 2012.

[6] M. A. Gernsbacher, A. R. Raimond, M. T. Balinghasay, and J. S. Boston, "Special need is an ineffective euphemism," Cognitive Research: Principles and Implications, vol. 1, no. 1, p. 29, 2016

[7] R. J. Berger, Introducing Disability Studies, Boulder: Lynne Reiner Publishers, 2013

[8] White paper on rights of persons with disabilities. (2015). [Online]. Available:

https://www.ru.ac.za/media/rhodesuniversity/content/equityinstitution alculture/documents/White\%20Paper\%20on\%20the\%20Rights\%20of $\% 20$ Persons\%20with\%20Disabilities.pdf

[9] D. S. Grewal, "A critical conceptual analysis of definitions of AI as applicable to computer," IOSR Journal of Computer Engineering, vol. 16, issue 2, pp. 9-13, 2014.

[10] C. Morrison, E. Cutrell, and A. Dhareshwar, "Imagining AI applications with people with visual disabilities using tactile ideation," in Proc. ASSETS '17, 2017.

[11] Lynch. (2018). How AI is improving assistive technology. [Online]. Available:

https://www.thetechedvocate.org/how-artificial-intelligence-is-improv ing-assistive-technology/

[12] Itsquiz. (2016). AI in special education. [Online]. Available: https://medium.com/@itsquiz15/artificial-intelligence-in-special-educ ation-dab27649b9b6

[13] J. Prentzas. (2013). AI methods in early childhood education. [Online] Available:

https://www.researchgate.net/publication/287644942_Artificial_Intell igence_Methods_in_Early_Childhood_Education

[14] Y. D. Kim, J. W. Hong, W. S. Kang, S. S. Baek, H. S. Lee, and J. An, "Design of robot assisted observation system for therapy and education of children with autism," in Proc. ICSR 2010, 2010.

[15] A. Allebee. (2017). Equadex, Microsoft cognitive services. [Online]. Available: https://customers.microsoft.com/en-us/story/equadex-partner-professi onal-services-cognitive-services

[16] J. Roach. (2018). AI technology helps students who are deaf learn. [Online].

Available: https://blogs.microsoft.com/ai/ai-powered-captioning/

[17] Sam McNeil. (2018). AI technology assisting deaf students. [Online]. Available:

https://educationblog.microsoft.com/2018/04/ai-technology-assistingdeaf-students/

[18] F.-G. Zeng, "A new landscape for hearing aids," The Hearing Journal, vol. 70, issue 12 , p. 6, 2017.

[19] N. S. Mboshi, "Teaching learners with visual impairment in an inclusive education setting: The cameroon perspective," International Journal of Education and Research, vol. 6, no. 2, February 2018

[20] UNICEF, "Teachers, inclusive, child-centred teaching and pedagogy," in Webinar 12 - Companion Technical Booklet, 2014.

Copyright $(\odot 2020$ by the authors. This is an open access article distributed under the Creative Commons Attribution License which permits unrestricted use, distribution, and reproduction in any medium, provided the original work is properly cited (CC BY 4.0)

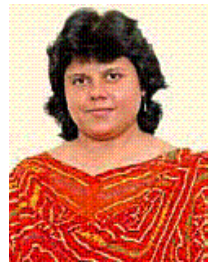

Shalini Garg is a prolific scholar with 20 years of academic experience in human resource management, organization behavior and organization development She is currently employed as a professor in University School of Management Studies, Guru Gobind Singh Indraprastha University, Delhi, India. She is intimately knowledgeable about HR aspects of Inclusion \& Accessibility and her teaching and research focuses on inclusion and accessibility and integration of $\mathrm{HR}$ Technology with social systems with an emphasis on emerging economies like India. She holds membership of Academy of Management and is on the editorial boards of National and International Journals. She has published extensively in numerous journals and presented her research to domestic and international audience. She has developed a Training Manual: Framework for HR Enabled Inclusion and Accessibility Training (An Overview) and also authored module on Organizational Inclusiveness - Gender, Special needs and Disability for Management School in IGNOU. She is an author of a book named Building Inclusive\& Accessible Workplaces, Emerald Publishing (2/12/2019). She is a project Director of Two Year IMPRESS ICSSR Project (2019-21) and was Prinicipal Investigator of Three Year MRP UGC Project (2015-2018). (For details: https://www.linkedin.com/in/dr-shalini-garg12345/).

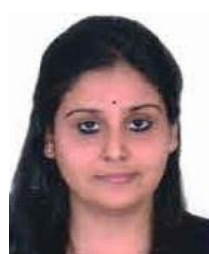

Shipra Sharma is presently working as an assistant professor in School of IT, CDAC, Noida, she has about 11 years of experience in the field of academia. Currently she's pursuing the Ph.D in management from GGSIP University, Dwarka. She has an experience in the area of Human Resource and Operations Management such as human resource management, human resource planning and development, human resource payroll, business communication and SAP (human resource management), operations management, total quality management, quality management system. 